\title{
Influence of musculoskeletal model parameter values on prediction of accurate knee contact forces during walking
}

\author{
Gil Serrancolí $^{\mathrm{a}}{ }^{*}$, Allison L. Kinney ${ }^{\mathrm{b}}$, Benjamin J. Fregly ${ }^{\mathrm{c}}$ \\ a Dept. of Mechanical Engineering, Universitat Politècnica de Catalunya, Av. Eduard Maristany 16, 08019 Barcelona, Catalunya, Spain \\ ${ }^{\mathrm{b}}$ Dept. of Mechanical \& Aerospace Engineering, University of Dayton, Dayton, OH 45469, United States \\ ${ }^{c}$ Dept. of Mechanical Engineering, Rice University, Houston, Texas 77005, United States
}

\section{ART ICLE INFO}

\section{Article history:}

Received 20 December 2019

Received in revised form 29 June 2020

Accepted 11 September 2020

Available online xxx

\section{Keywords}

Muscle-tendon model parameters

Muscle force estimation

Knee contact force prediction

Musculoskeletal modeling

\begin{abstract}
A B S T R A C T
Treatment design for musculoskeletal disorders using in silico patient-specific dynamic simulations is becoming a clinical possibility. However, these simulations are sensitive to model parameter values that are difficult to measure experimentally, and the influence of uncertainties in these parameter values on the accuracy of estimated knee contact forces remains unknown. This study evaluates which musculoskeletal model parameters have the greatest influence on estimating accurate knee contact forces during walking. We performed the evaluation using a two-level optimization algorithm where musculoskeletal model parameter values were adjusted in the outer level and muscle activations were estimated in the inner level. We tested the algorithm with different sets of design variables (combinations of optimal muscle fiber lengths, tendon slack lengths, and muscle moment arm offsets) resulting in nine different optimization problems. The most accurate lateral knee contact force predictions were obtained when tendon slack lengths and moment arm offsets were adjusted simultaneously, and the most accurate medial knee contact force estimations were obtained when all three types of parameters were adjusted together. Inclusion of moment arm offsets as design variables was more important than including either tendon slack lengths or optimal muscle fiber lengths alone to obtain accurate medial and lateral knee contact force predictions. These results provide guidance on which musculoskeletal model parameter values should be calibrated when seeking to predict in vivo knee contact forces accurately.
\end{abstract}

\section{Introduction}

Roughly $10 \%$ of the European population over age 60 is affected by osteoarthritis [1], with the knee being one of the joints most commonly affected. Those with knee osteoarthritis often suffer pain and loss of function [2], which in turn affects their ability to perform activities of daily living. While increased age is a contributing factor for knee osteoarthritis, the causes and evolution of this disease are not yet fully understood [3]. Though treatment of knee osteoarthritis could be improved if knee contact forces could be predicted accurately for individual subjects, the ability to generate reasonably accurate predictions remains an open research question [4], especially when medial and lateral knee contact forces are predicted simultaneously [5].

Few studies have measured in vivo knee contact forces due to the invasiveness of performing such measurements [6-9], and the force measurement in healthy subjects and subjects with osteoarthritis remains a currently intractable challenge. Consequently, knee contact forces are most commonly estimated using dynamic musculoskeletal models that relate measured human body movement and external forces to generated internal forces. External forces determine the

\footnotetext{
* Correspondence address.

E-mail address: gil.serrancoli@upc.edu (G. Serrancolí)
}

net superior-inferior force and varus-valgus moment that must be balanced by internal forces from muscles, articular contact, and ligaments. For a given external loading pattern, muscles will be the main determinants of knee contact forces [10]. Therefore, the estimation of knee contact forces typically involves estimation of leg muscle forces, which in turn requires solving an indeterminate problem typically using optimization [11-15] or EMG-driven modeling [16-19] methods. The main difference between optimization and EMG-driven methods for estimating muscle forces is that EMG-driven methods calibrate key model parameter values (e.g., optimal muscle fiber length, tendon slack length) using the subject's EMG and motion data, while for optimization methods, EMG data are not used and model parameter values are rarely calibrated. Since leg muscle forces also cannot be measured non-invasively in vivo under clinical conditions, validation of knee contact forces estimated by dynamic musculoskeletal models remains a challenge.

To complicate matters further, knee contact and leg muscle forces estimated by musculoskeletal models are sensitive to model parameter values that are difficult to calibrate using commonly available experimental data. Several studies have explored the sensitivity of leg muscle force estimates to uncertainties in musculoskeletal model parameter values [20-27]. In contrast, few studies have investigated the sensitivity of estimated knee contact forces to uncertainties in model parameter values. Valente et al. [28] concluded that uncertainties in body landmark positions, musculoskeletal geometry, and maximum mus- 
cle tension could lead to differences in predicted total knee contact forces up to 2.1 times body weight. Similar conclusions (although with a lower variability in total knee contact forces) were obtained by Navacchia et al. [29]. Lund et al. [30] suggested that the calibration of the positions and orientation of the lower body joints would result in more accurate predictions of total knee contact force. Heller et al. [31] also analyzed how the axial alignment influenced the knee contact forces. However, it remains unknown which musculoskeletal model parameter values affect the model's ability to predict accurate medial and lateral knee contact forces.

This study assesses which musculoskeletal model parameters have the greatest influence on predicting knee contact force accurately for walking. To this end, we developed a two-level optimization approach that adjusted constant muscle-related parameter values in the outer level and time-varying muscle activations in the inner level. The goal was for the outer-level optimization to find model parameter values that would cause the inner-level optimization to match experimentally measured medial and lateral knee contact forces [6] without including them anywhere in the inner-level problem formulation. By using different sets of outer-level design variables composed of different combinations of muscle-tendon and geometric parameters, we identified which parameters should be included as design variables in the outer level optimization to obtain accurate knee contact force predictions.

\section{Methods}

\subsection{Experimental data}

Experimental data for our study were obtained from the fourth Grand Challenge Competition to Predict In Vivo Knee Loads [6]. The data consisted of surface marker trajectories, ground reaction forces, single-plane fluoroscopic knee motion trajectories, and in vivo knee contact forces for a subject implanted with a force-measuring tibial prosthesis (gender: male, age: 88 years, mass: $65 \mathrm{~kg}$, height: $166 \mathrm{~cm}$, implanted knee: right). The prosthesis had four uniaxial load cells located in the four quadrants of the tibial tray [8]. The data used for the study included six normal overground gait trials performed at the subject's self-selected speed $(1.26 \pm 0.03 \mathrm{~m} / \mathrm{s})$. Ground reaction forces from three force plates and in vivo knee contact forces were processed using standard methods (low-pass filtered at $6 \mathrm{~Hz}$ using a fourth-order zero phase-lag Butterworth filter).

\subsection{Musculoskeletal model}

A subject-specific OpenSim [32,33] musculoskeletal model of the lower body (pelvis, thigh, shank, and foot) possessing 44 muscle-tendon units per leg (see Table S.1 for muscle names) was used for this study, as in previous work by members of our group [34]. The model incorporated subject-specific pelvis and lower body bone models constructed from subject CT scan data. Peak isometric force values, optimal muscle fiber length and tendon slack length values were derived from the works by Arnold et al. ([35,36]) and scaled following an approach similar to Campen et al. [26] to avoid infeasible initial guesses (Table S.1.). The left leg of the scaled model was removed, and the kinematic structure of the scaled model was modified to possess 23 degrees of freedom (DOFs): three translations and three rotations defining the position and orientation of the pelvis with respect to ground, three rotations (flexion, adduction, and rotation) for the hip, three rotations (flexion, adduction, and rotation) and three translations (superior-inferior, anterior-posterior, and medial-lateral) for the knee, three rotations (flexion, adduction, and rotation) and three translations (superior-inferior, anterior-posterior, and medial-lateral) for the patella relative to the femur, and two rotations (flexion and eversion) for the ankle. Patellar flexion motion was prescribed as a function of knee angle, as in our earlier work [34], and the other patellar degrees of freedom were locked to constant values.
The OpenSim model was used to calculate joint kinematics, inverse dynamic joint loads, muscle-tendon kinematics and moment arms, and ultimately knee contact forces for each gait trial using the available experimental data. Detailed knee kinematics were obtained by combining fluoroscopy, marker motion, and knee contact force data using pose estimation analyses performed with an elastic foundation contact model. Given the knee flexion angle time history from an initial inverse kinematics analysis, and internal-external rotation and anterior-posterior translation time histories from fluoroscopy data, the pose estimation algorithm estimated the superior-inferior and medial-lateral translation and varus-valgus rotation time histories. This algorithm matched the experimental medial and lateral knee contact forces while minimizing the mediolateral contact force. A more detailed description can be found elsewhere [37]. Remaining joint kinematics of the model were calculated using OpenSim inverse kinematics analyses. Net loads acting at the lower body joints were calculated using OpenSim inverse dynamics analyses, where the input joint kinematics were low-pass filtered at $6 \mathrm{~Hz}$ (four-order zero phase-lag Butterworth filter). Muscle-tendon lengths, velocities, and moment arms were calculated using OpenSim muscle analyses. Once muscle forces were estimated using the two-level optimization approach described below, muscle force contributions to the net knee superior-inferior force and the net knee adduction moment were obtained using the calculated moment arms. Knee contact force contributions to the same two net knee loads were estimated by subtracting the calculated muscle force contributions from the net loads, where ligament contributions to net knee loads were assumed to be zero, as in previous work by our group [34]. Medial and lateral knee contact forces were then calculated from the superior-inferior knee contact force and varus-valgus knee contact moment using validated regression relationships reported by Zhao and co-workers [38].

\subsection{Optimization problem formulation}

A two-level static optimization procedure was developed in Matlab (The Mathworks, Natick, MA) to analyze how the accuracy of model-predicted medial and lateral knee contact forces were affected by the calibration of different types of model parameter values (i.e., optimal muscle fiber lengths, tendon slack lengths, and moment arm offsets). The outer level used Matlab's trust region reflective nonlinear least-squares algorithm [39] to adjust specified types of model parameter values so as to match the experimentally measured medial and lateral knee contact forces as closely as possible. Design variables for optimal muscle fiber lengths and tendon slack lengths were scale factors (one per muscle) that multiplied the scaled literature values, while design variables for moment arm offsets (one per muscle per spanned joint) were constant values added to the moment arms obtained from OpenSim. The cost function used quadratic error terms to track the in vivo medial and lateral knee contact forces, made maximum normalized muscle fiber lengths close to one (following the findings of Arnold and Delp [40]), minimized reserve activations from the inner level, minimized scale factor differences between optimal muscle fiber length and tendon slack length for each muscle, and minimized moment arm offsets (see Section S.2 for a detailed formulation).

Given the current guess for model parameter values from the outer level (muscle fiber lengths, tendon slack lengths and muscle moment arms offsets), the inner level optimization used Matlab's quadratic programming algorithm to minimize squared muscle and reserve activations so as to match six net loads from inverse dynamics (3 hip moments, 1 knee moment for flexion-extension, and 2 ankle moments) (Section S.3). The contact force contributions to those loads were considered to be negligible. Design variables were time-varying muscle activations along with reserve activations. The cost function used quadratic terms to minimize both muscle and reserve activations. Linear equality constraints were used to match the six net loads from inverse dynamics. Muscle forces were calculated using a Hill-type muscle-ten- 
don model with rigid tendon developed in Matlab and possessing continuous force-length and force-velocity properties (see De Groote et al. [41] for details). No knee contact force information was used in the inner level, and the muscle activations estimated by the inner level were passed to the outer level for calculating the resulting medial and lateral knee contact forces. Note that knee adduction and superior-inferior moment arms were only used to estimate knee contact forces and were not used in the inner level optimization.

The accuracy of predicted knee contact forces when different types of model parameter values were calibrated was investigated using a two-step process. First, the complete two-level optimization was run using three gait trials together to calibrate the specified types of model parameters. The output of this optimization was a calibrated model which, when used in the inner-level optimization, would estimate medial and lateral knee contact forces as closely as possible for the three selected gait trials (calibration trials). Second, only the inner-level optimization was run using three additional gait trials to test the accuracy with which the previously calibrated model could predict medial and lateral knee contact forces using non-calibration walking trials (prediction trials). The complete code can be found in the following link: https://github.com/gilserrancoli/mskkneeforces.

Table 1

Musculoskeletal parameters values considered in each problem formulation. DV stands for design variables.

\begin{tabular}{llll}
\hline $\begin{array}{l}\text { Problem } \\
\text { number }\end{array}$ & Musculoskeletal parameters values & \\
\hline & $\begin{array}{l}\text { Scale factors of optimal } \\
\text { fiber lengths }\end{array}$ & $\begin{array}{l}\text { Scale factors of tendon } \\
\text { slack lengths }\end{array}$ & $\begin{array}{l}\text { Moment arm } \\
\text { deviations }\end{array}$ \\
\hline 1 & DV & 1 & 0 \\
2 & 1 & DV & 0 \\
3 & 1 & 1 & DV \\
4 & DV & DV & 0 \\
5 & DV & 1 & DV \\
6 & 1 & DV & DV \\
$7,8,9$ & DV & DV & DV \\
\hline
\end{tabular}

Table 2

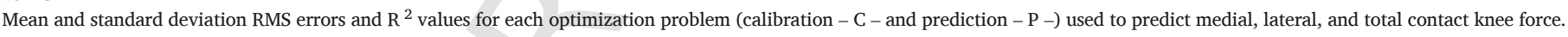

\begin{tabular}{|c|c|c|c|c|c|c|c|}
\hline \multirow[t]{2}{*}{ Problem number } & \multirow[t]{2}{*}{ Type } & \multicolumn{3}{|c|}{ RMS errors (mean \pm std) } & \multicolumn{3}{|c|}{$\mathrm{R}^{2}$ values (mean \pm std) } \\
\hline & & Medial & Lateral & Total & Medial & Lateral & Total \\
\hline \multirow[t]{2}{*}{1} & $\mathrm{C}$ & $104.5 \pm 78.3$ & $151.7 \pm 32.9$ & $209.1 \pm 59.1$ & $0.90 \pm 0.70$ & $0.08 \pm 0.12$ & $0.82 \pm 0.54$ \\
\hline & $\mathrm{P}$ & $133.0 \pm 40.3$ & $153.6 \pm 41.8$ & $201.2 \pm 34.0$ & $0.84 \pm 0.19$ & $0.25 \pm 0.46$ & $0.82 \pm 0.36$ \\
\hline \multirow[t]{2}{*}{2} & $\mathrm{C}$ & $102.2 \pm 75.0$ & $137.7 \pm 37.0$ & $201.4 \pm 55.2$ & $0.90 \pm 0.62$ & $0.23 \pm 0.08$ & $0.83 \pm 0.40$ \\
\hline & $\mathrm{P}$ & $129.1 \pm 44.5$ & $141.5 \pm 46.0$ & $200.1 \pm 28.3$ & $0.85 \pm 0.19$ & $0.36 \pm 0.39$ & $0.82 \pm 0.25$ \\
\hline \multirow[t]{2}{*}{3} & $\mathrm{C}$ & $75.9 \pm 29.9$ & $103.7 \pm 49.4$ & $161.9 \pm 60.4$ & $0.95 \pm 0.10$ & $0.57 \pm 0.13$ & $0.88 \pm 0.39$ \\
\hline & $\mathrm{P}$ & $108.5 \pm 28.3$ & $141.4 \pm 47.6$ & $197.9 \pm 61.6$ & $0.89 \pm 0.22$ & $0.37 \pm 0.39$ & $0.83 \pm 0.25$ \\
\hline \multirow[t]{2}{*}{4} & $\mathrm{C}$ & $99.6 \pm 64.7$ & $126.0 \pm 40.3$ & $190.7 \pm 54.8$ & $0.91 \pm 0.48$ & $0.35 \pm 0.05$ & $0.85 \pm 0.40$ \\
\hline & $\mathrm{P}$ & $114.2 \pm 45.2$ & $141.2 \pm 42.7$ & $191.3 \pm 31.5$ & $0.88 \pm 0.23$ & $0.35 \pm 0.44$ & $0.83 \pm 0.22$ \\
\hline \multirow[t]{2}{*}{5} & $\mathrm{C}$ & $66.6 \pm 25.0$ & $74.7 \pm 27.2$ & $119.7 \pm 40.4$ & $0.96 \pm 0.08$ & $0.78 \pm 0.03$ & $0.94 \pm 0.19$ \\
\hline & $\mathrm{P}$ & $100.6 \pm 24.6$ & $112.3 \pm 30.9$ & $168.6 \pm 60.4$ & $0.91 \pm 0.06$ & $0.58 \pm 0.34$ & $0.87 \pm 0.15$ \\
\hline \multirow[t]{2}{*}{6} & $\mathrm{C}$ & $69.1 \pm 28.9$ & $69.8 \pm 35.4$ & $124.4 \pm 39.0$ & $0.95 \pm 0.06$ & $0.81 \pm 0.02$ & $0.93 \pm 0.16$ \\
\hline & $\mathrm{P}$ & $115.2 \pm 18.5$ & $93.8 \pm 13.2$ & $146.6 \pm 52.9$ & $0.88 \pm 0.08$ & $0.70 \pm 0.23$ & $0.91 \pm 0.04$ \\
\hline \multirow[t]{2}{*}{7} & $\mathrm{C}$ & $59.9 \pm 30.4$ & $73.2 \pm 26.8$ & $115.2 \pm 36.9$ & $0.97 \pm 0.07$ & $0.79 \pm 0.05$ & $0.94 \pm 0.17$ \\
\hline & $\mathrm{P}$ & $93.9 \pm 11.2$ & $109.8 \pm 26.6$ & $148.8 \pm 49.5$ & $0.92 \pm 0.11$ & $0.60 \pm 0.30$ & $0.90 \pm 0.12$ \\
\hline \multirow[t]{2}{*}{8} & $\mathrm{C}$ & $69.6 \pm 29.8$ & $102.0 \pm 44.9$ & $152.8 \pm 54.2$ & $0.95 \pm 0.11$ & $0.59 \pm 0.16$ & $0.89 \pm 0.30$ \\
\hline & $\mathrm{P}$ & $92.6 \pm 27.0$ & $178.8 \pm 43.3$ & $186.7 \pm 87.5$ & $0.92 \pm 0.44$ & $-0.01 \pm 0.65$ & $0.85 \pm 0.46$ \\
\hline \multirow[t]{2}{*}{9} & $\mathrm{C}$ & $72.6 \pm 29.4$ & $61.1 \pm 39.4$ & $121.1 \pm 30.7$ & $0.95 \pm 0.04$ & $0.86 \pm 0.02$ & $0.93 \pm 0.10$ \\
\hline & $\mathrm{P}$ & $121.5 \pm 3.5$ & $118.8 \pm 28.6$ & $167.1 \pm 51.1$ & $0.87 \pm 0.24$ & $0.56 \pm 0.17$ & $0.88 \pm 0.13$ \\
\hline
\end{tabular}

\subsection{Analyses}

Nine different "calibrate-then test" problems were performed to evaluate how knee contact force prediction accuracy was affected by the calibrated types of model parameters (see Table 1). Unless otherwise noted, initial conditions for each problem were taken from the scaled OpenSim model described above. Problems 1 to 3 optimized only one type of design variable (muscle fiber lengths, tendon slack lengths, and moment arm offsets, respectively) and kept the other possible variables constant. Problems 4 to 6 used two types of parameters as design variables, as outlined in Table 1. Problem 7 used all three types of parameters as design variables. Problem 8 was a variant of Problem 7 where a common scale factor was used for optimal muscle fiber length and tendon slack length for each muscle. Problem 9 was also a variant of Problem 7 where the tracking weight for lateral knee contact force was increased by $10 \%$ and the tracking weight for medial knee contact force was decreased by $10 \%$, since lateral contact force is often more difficult to predict accurately than is medial contact force [5]. Different problems used different initial guesses to make use of the best information available at the start of each problem (see Section S.4 from Supplementary Material).

For each problem, root mean square (RMS) errors and coefficient of determination $\left(R^{2}\right)$ values were calculated to compare the differences in magnitude and shape, respectively, between the estimated medial and lateral knee contact forces and their experimental values.

\section{Results}

Medial knee contact force was well matched in both shape and magnitude for all optimization problems in calibration (mean $\mathrm{R}^{2} \geq 0.9$ and mean RMS $<105.0 \mathrm{~N}$ ) and prediction (mean $\mathrm{R}^{2} \geq 0.84$ and mean RMS $<133.0 \mathrm{~N}$ ) trials (Table 2). Lateral contact force was overall better matched in calibration and prediction trials when moment arm deviations were included as design variables (Problems 3 and 5 to 7), especially in terms of shape. For both calibration and prediction trials, the most accurate matching of medial force was obtained when varying all three types of design variables (Problem 7), whereas the most accurate matching of lateral force was obtained when varying only tendon slack lengths and moment arm deviations (Problem 6). Optimal solutions were only slightly influenced by initial guess. When different ini- 
tial guesses were used, differences in RMS values of knee contact force for calibration trials were less than $10 \mathrm{~N}$.

Optimization problems that varied only optimal fiber lengths (Problem 1) or tendon slack lengths (Problem 2) led to the poorest re- sults (Figs. 1-4 and Table 2). Estimated contact forces were similar for these two problems, with RMS differences between the two solutions for calibration and prediction trials being, on average, $22.3 \mathrm{~N}$ and $7.0 \mathrm{~N}$, respectively, for medial contact force and $35.8 \mathrm{~N}$ and $11.8 \mathrm{~N}$, re-
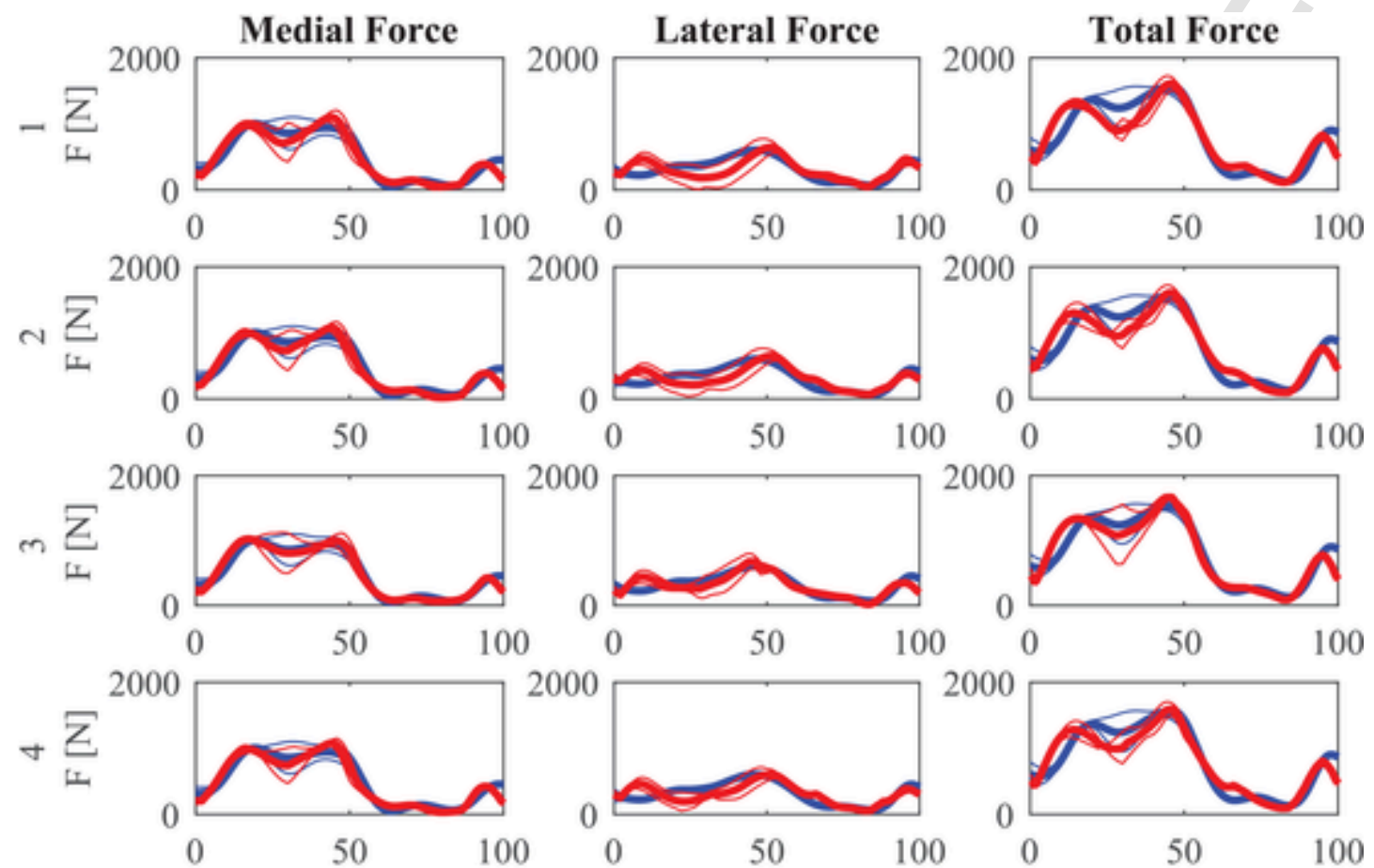

2000

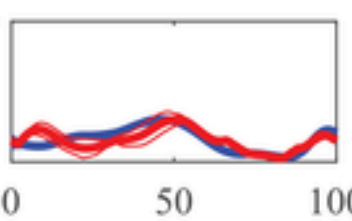

2000
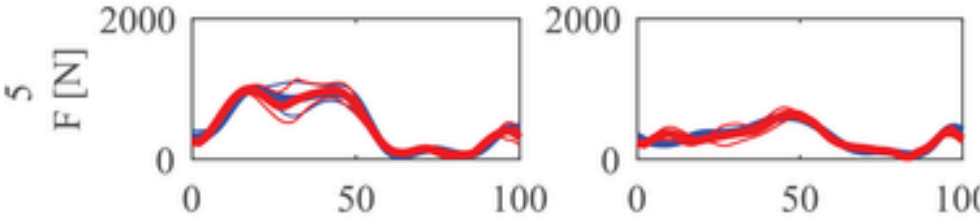

$100 \quad 0$
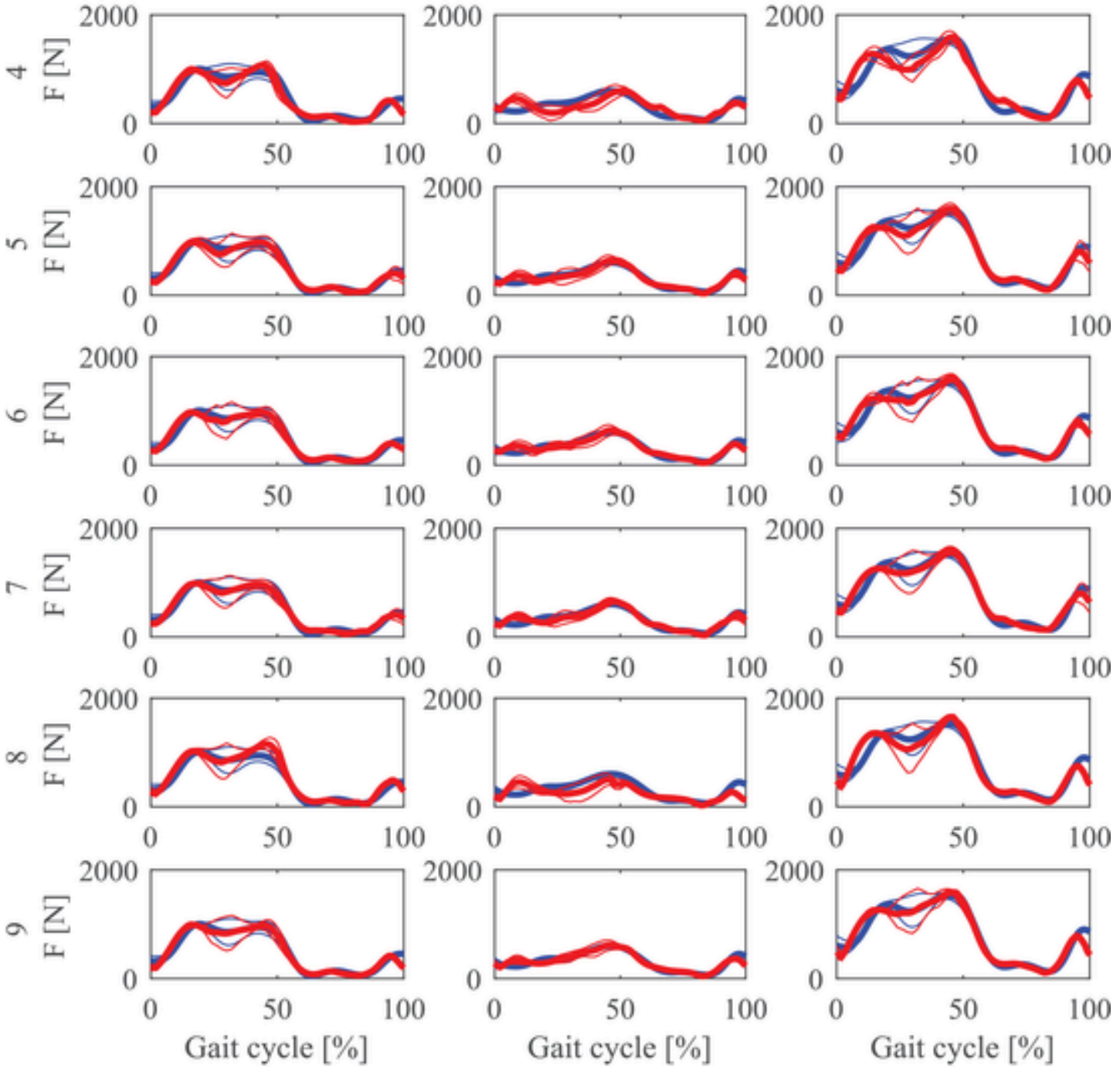

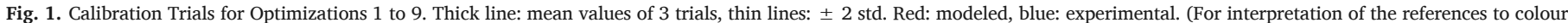
in this figure legend, the reader is referred to the web version of this article.) 


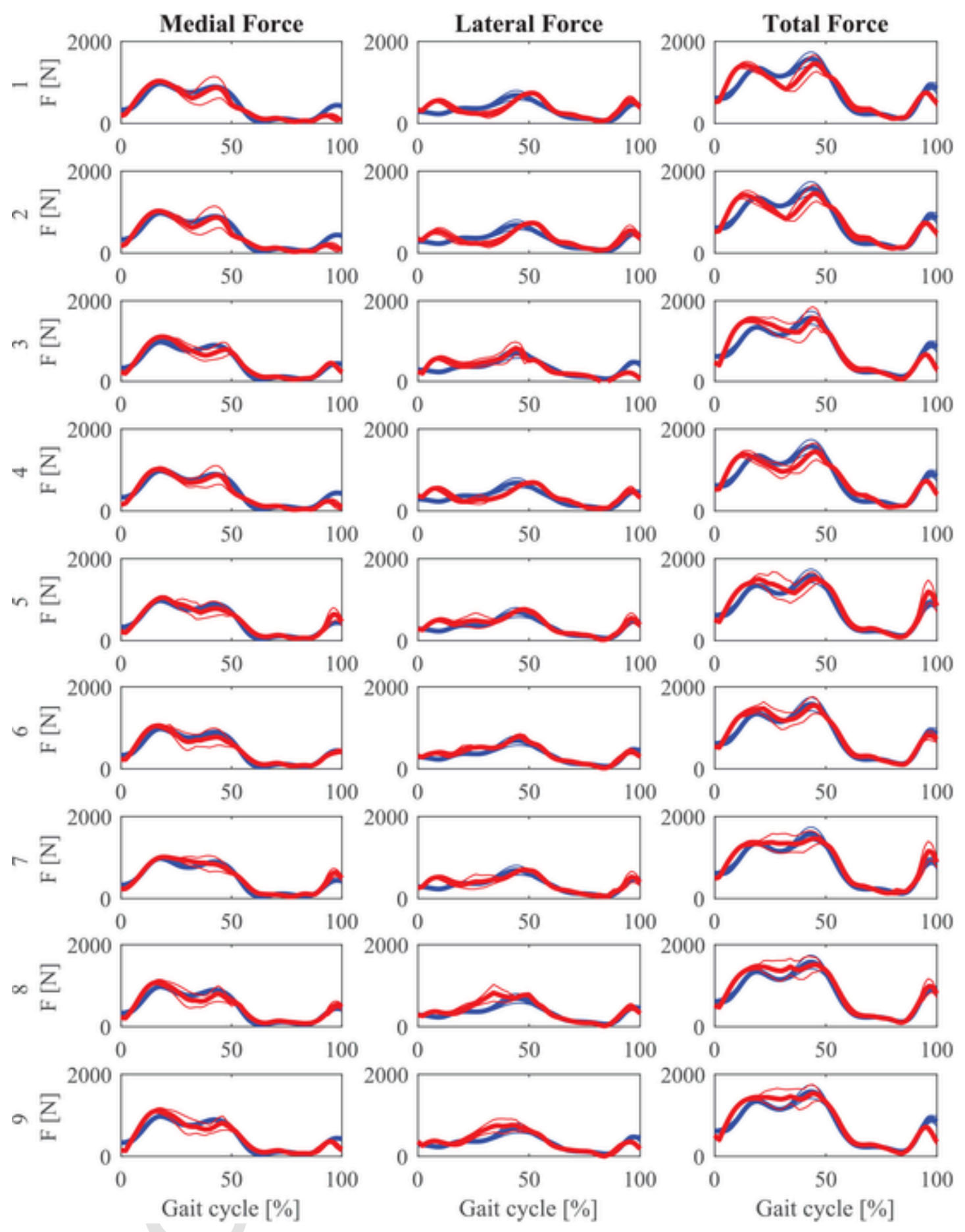

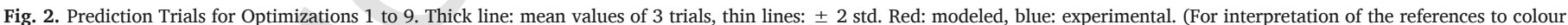
in this figure legend, the reader is referred to the web version of this article.)

spectively, for lateral contact force. Both optimizations overpredicted the second peak of medial contact force in stance phase (at about 50\% of the gait cycle). When moment arm deviations alone were optimized (Problem 3), total contact force was matched better than in Problems 1 and 2, especially for calibration trials (Table 2). However, lateral contact force for prediction trials was not matched better than in Problems 1 and 2 (mean RMS error $=141.4 \mathrm{~N}$ and mean $\mathrm{R}^{2}$ value $=0.37$ ). In Problems 1 to 3, estimated lateral contact force had a non-realistic peak at about $10 \%$ of the gait cycle.
The combination of moment arm deviations with optimal fiber lengths (Problem 5) or tendon slack lengths (Problem 6) as design variables led to improvements in contact force matching. Optimization of optimal fiber lengths and tendon slack lengths together (Problem 4) did not improve knee contact force predictions with respect to Problem 3. For calibration trials, Problem 4 also overpredicted the second peak of medial force during stance phase. For calibration and prediction trials, Problem 5 matched medial forces better, and lateral contact forces worse, than in Problem 6. Problem 5 matched total contact force better for calibration trials, whereas Problem 6 matched it better for predic- 


\section{Calibration trials}

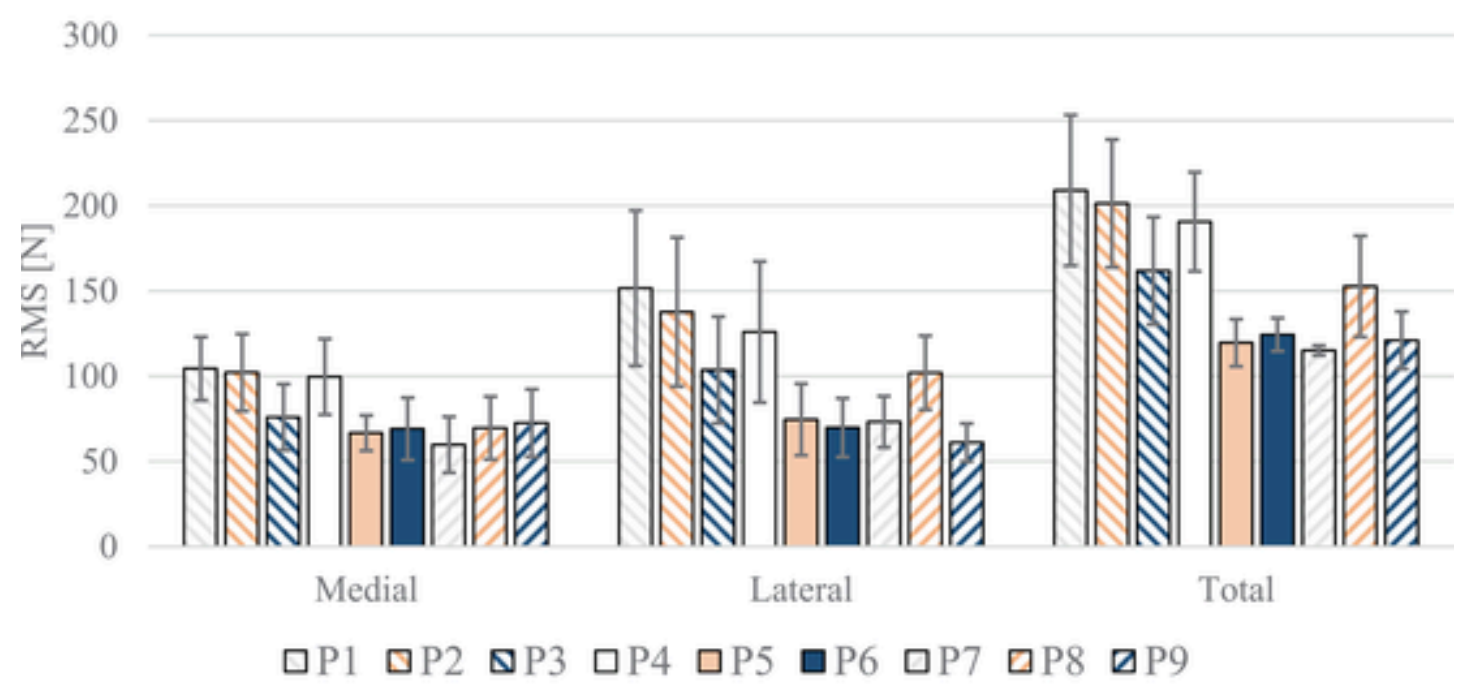

\section{Prediction trials}

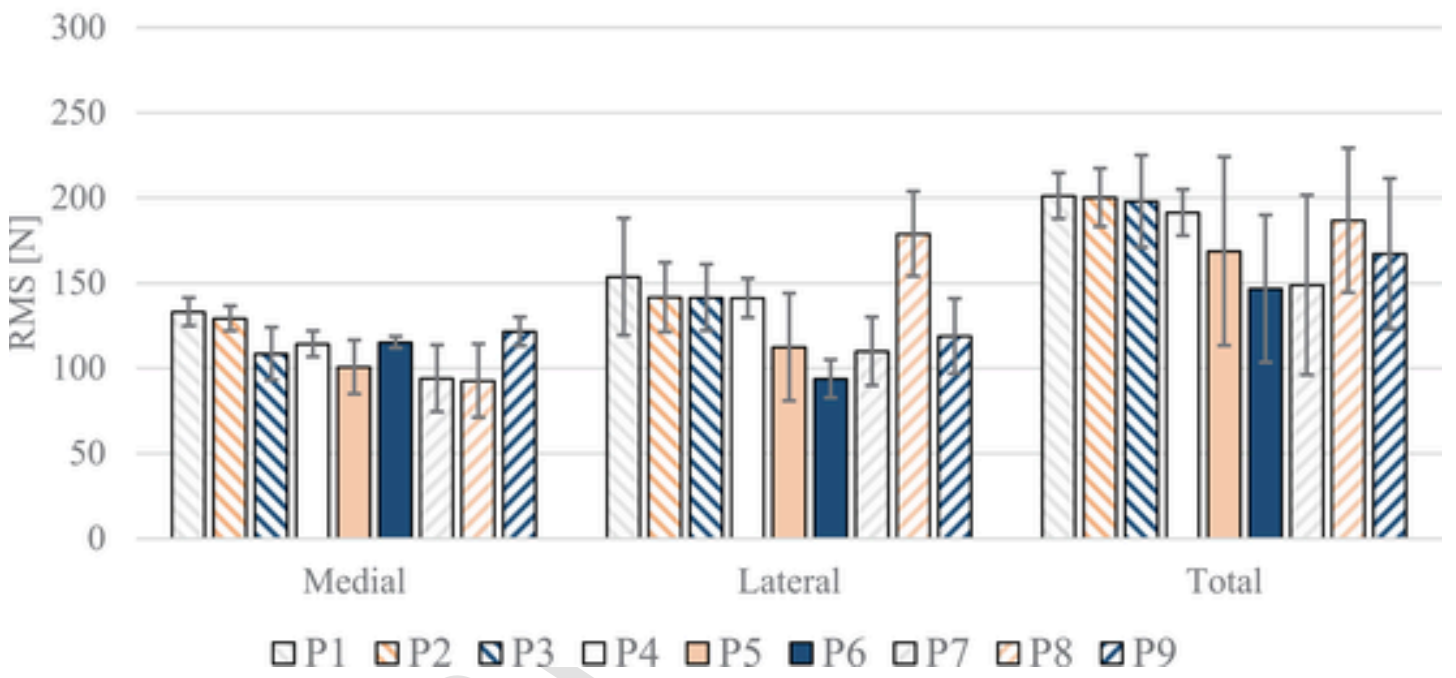

Fig. 3. Mean and standard deviation of the RMS errors for calibration (above) and prediction (below) trials.

tion trials. Both Problems 5 and 6 decreased the erroneous lateral force peak at $10 \%$ of the gait cycle observed in Problems 1 to 4 .

The most accurate tracking of medial contact force was obtained when varying all three types of parameters together (Problem 7). However, the results of this problem were not better than in Problem 6 for lateral contact force, which continued to possess the unrealistic peak at the beginning of stance phase. When the outer-level cost function weight was increased by $10 \%$ for lateral contact force tracking and decreased by $10 \%$ for medial contact force tracking (Problem 9), the matching of lateral contact force for calibration trials was improved (from mean RMS error $=73.2 \pm 26.8 \mathrm{~N}$ and mean $R^{2}$ value $=0.79 \pm 0.05$ in Problem 7 to mean RMS error $=61.1 \pm 39.4 \mathrm{~N}$ and mean $\mathrm{R}^{2}$ value $=0.86 \pm 0.02$ in Problem 9) while matching of medial contact force worsened slightly (from mean RMS error $=59.9 \pm 30.4 \mathrm{~N}$ and mean $R^{2}$ value $=0.97 \pm 0.07$ in Problem 7 to mean RMS error $=72.6 \pm 29.4 \mathrm{~N}$ and mean $R^{2}$ value $=0.95 \pm 0.04$ in Problem 9). Furthermore, tracking of both medial and lateral contact forces worsened for prediction trials (from medial: mean RMS error $=93.9 \pm 11.2 \mathrm{~N}$ and mean $\mathrm{R}^{2}$ value $=0.92 \pm 0.11$, lateral: mean RMS and mean $\mathrm{R}^{2}$ value $=0.60 \pm 0.30$ in Problem 7; to medial: mean RMS error $=121.5 \pm 3.5 \mathrm{~N}$ and mean $R^{2}$ value $=0.87 \pm 0.24$, lateral: mean RMS error $=118.8 \pm 28.6 \mathrm{~N}$ and mean $\mathrm{R}^{2}$ value $=0.56 \pm 0.17$ in Problem 9).

Using a common scale factor for optimal fiber lengths and tendon slack lengths along with moment arm deviations (Problem 8) did not improve the results compared to Problems 5 to 7. Specifically, results were not better for either medial and lateral contact force in calibration trials or lateral contact force in prediction trials (in which the RMS errors were increased more than $60 \mathrm{~N}$ ). The variation of these two types of parameter values (fewer design variables than in Problem 7) was small (less than 3\%) compared to the nominal values in Problems 5 to 7. The variation of optimal fiber lengths with respect to literature values was $7.4 \pm 15.2 \%$ and $7.2 \pm 21.5 \%$ for Problems 5 and 7 , respectively, and the variation of tendon slack lengths was $10.2 \pm 12.3 \%$ and $5.6 \pm 19.8 \%$ for Problems 6 and 7, respectively (Fig. 5).

Muscle force contributions to medial and lateral knee contact force were different for some muscles among the different optimization problems. For example, Problem 1 and 7, GasMed and Semiten contribu- 


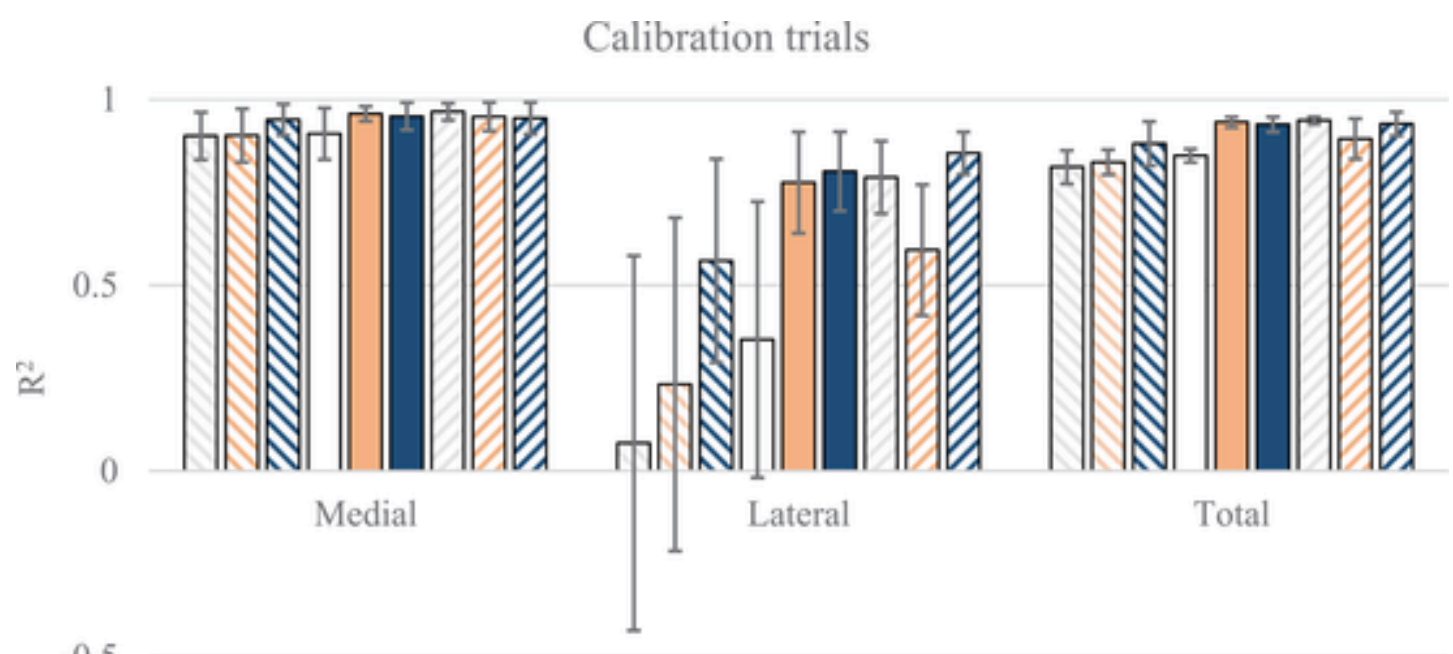

\section{$\square P 1 \square P 2 \otimes P 3 \quad \square P 4 \quad \square P 5 \square P 6 \square P 7 \square P 8 \square P 9$}

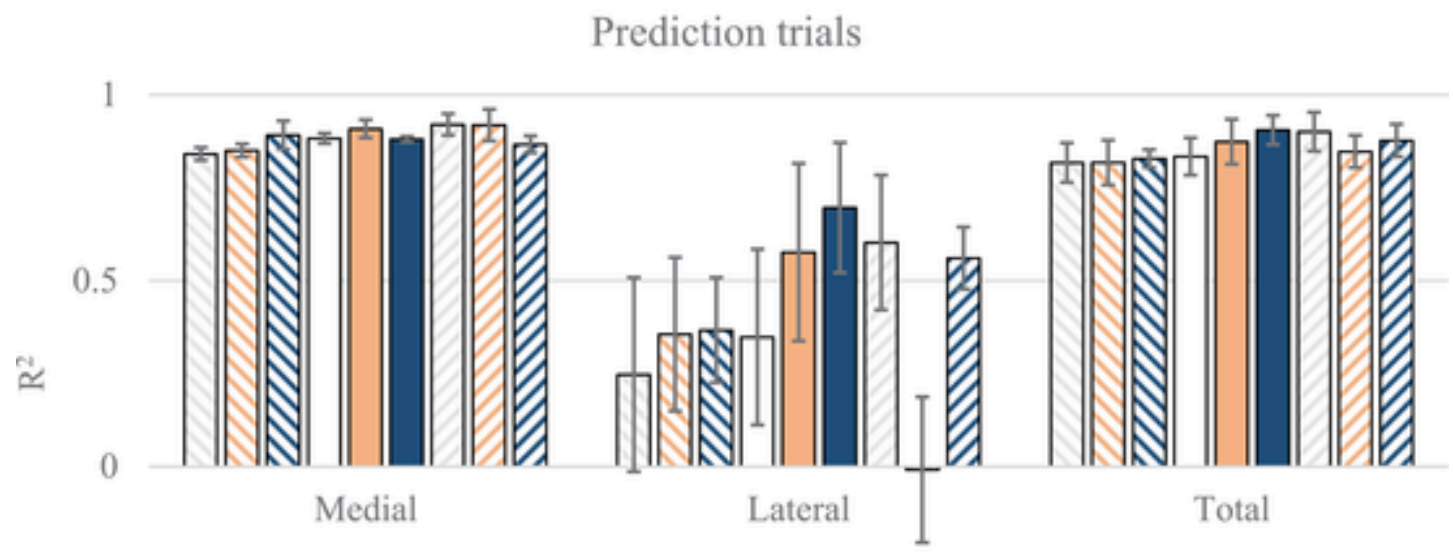

$-0.5$

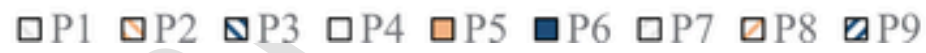

Fig. 4. Mean and standard deviation of the $\mathrm{R}^{2}$ values for calibration (above) and prediction (below) trials.

tions to medial contact force differed by around $100 \mathrm{~N}$ during stance phase (Fig. 6), while VasLat, BFSH and GasMed contributions to lateral contact differed by more than $100 \mathrm{~N}$ (Fig. 7). Muscle moment arm deviations were lower than $1 \mathrm{~cm}$ for all muscles (Fig. 8).

The nested optimization takes between $25 \mathrm{~min}$ (when optimizing only one set of parameters) to 95 min (when optimizing all three sets of parameters). The inner level optimization which can be used to predict muscle activations from previously calibrated musculotendon parameters takes just $0.09 \mathrm{~s}$ to run.

\section{Discussion}

This study evaluated which common musculoskeletal model parameters have the largest influence on predicting knee contact forces accurately during walking (at both medial and lateral compartments). A two-level static optimization procedure was used to calibrate musculoskeletal parameter values (time independent) in the outer level and predict muscle activations (time dependent) in the inner level. Comparison of modeled with experimental knee contact forces allowed indirect evaluation of whether the estimated leg muscle forces were realistic. The differences among the nine optimization problem formulations in- volved which types of parameters (optimal fiber lengths, tendon slack lengths, and/or moment arm deviations) were allowed to vary. Our results showed that inclusion of moment arm deviations as design variables improved calibration of the musculoskeletal model, since it led to more accurate knee contact force predictions. Optimizing only optimal fiber lengths and tendon slack lengths overpredicted the second peak of medial contact force during stance phase. These results provide insight into how calibration of different sets of muscle-related parameter values affect the accuracy of medial and lateral knee contact force predictions made using musculoskeletal models.

Published studies have reported contradictory results on the sensitivity of muscle force predictions to errors in muscle-tendon model parameter and moment arm values. On the one hand, some studies support the idea that the calibration of moment arms does not have a significant impact on estimated muscle function [42], even though moment arm differences can be large when comparing generic with patient-specific models [43]. Modenese et al. [44] analyzed differences in knee contact force predictions where optimal fiber lengths and tendon slack lengths were scaled with a constant ratio versus when they were scaled without altering normalized fiber length. The main differ- 


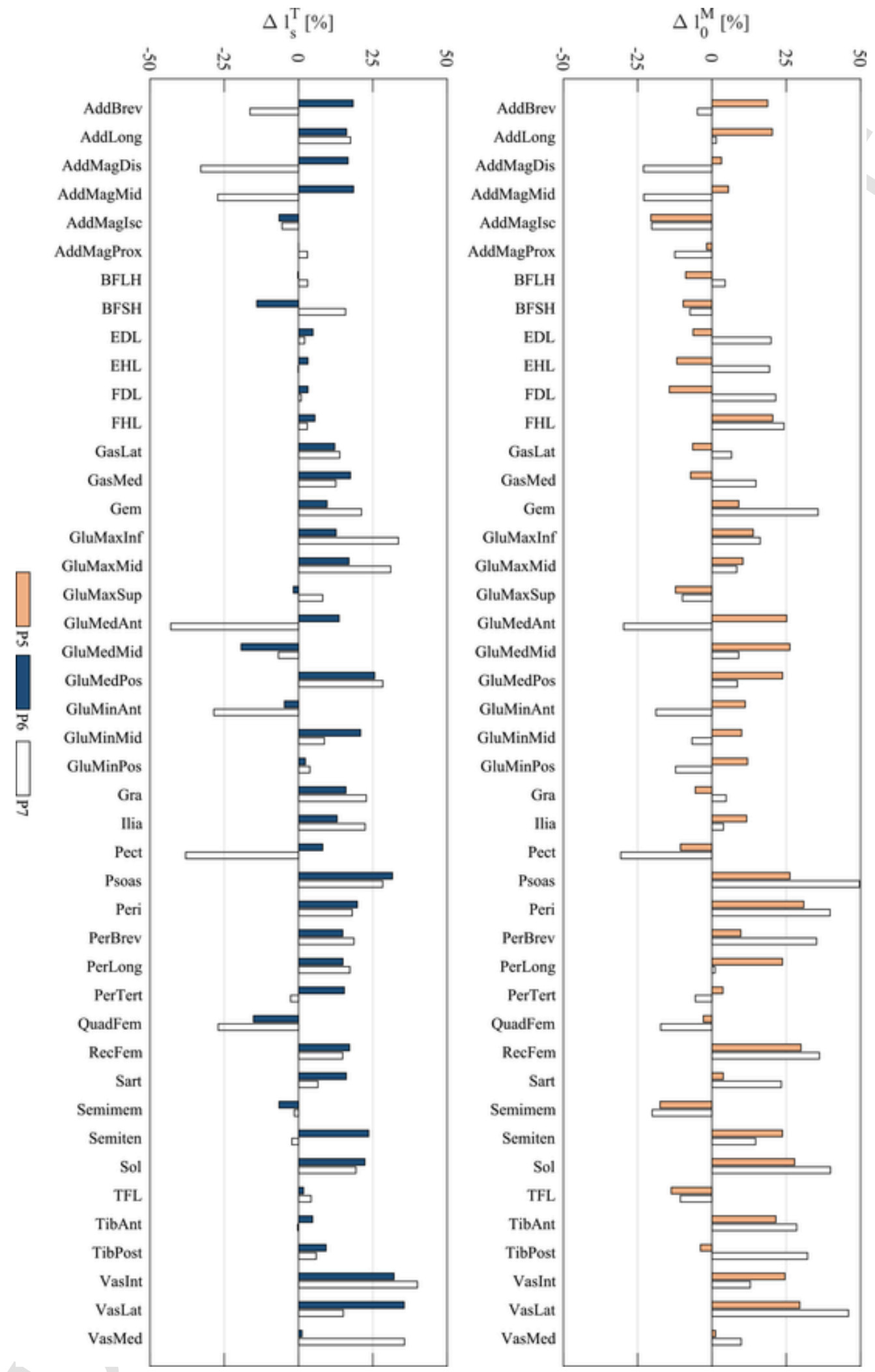

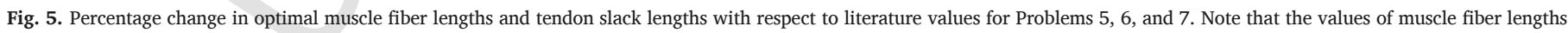
in Problem 6 and the values of tendon slack lengths in Problem 5 were not changed and equal to the literature values.

ences were in the second peak of total knee contact force during stance phase, which was more realistic when the normalized force-length curve was not altered.
On the other hand, other authors performed sensitivity analyses using Monte-Carlo methods to investigate which parameters affect muscle force predictions the most. The previous study most similar to ours 

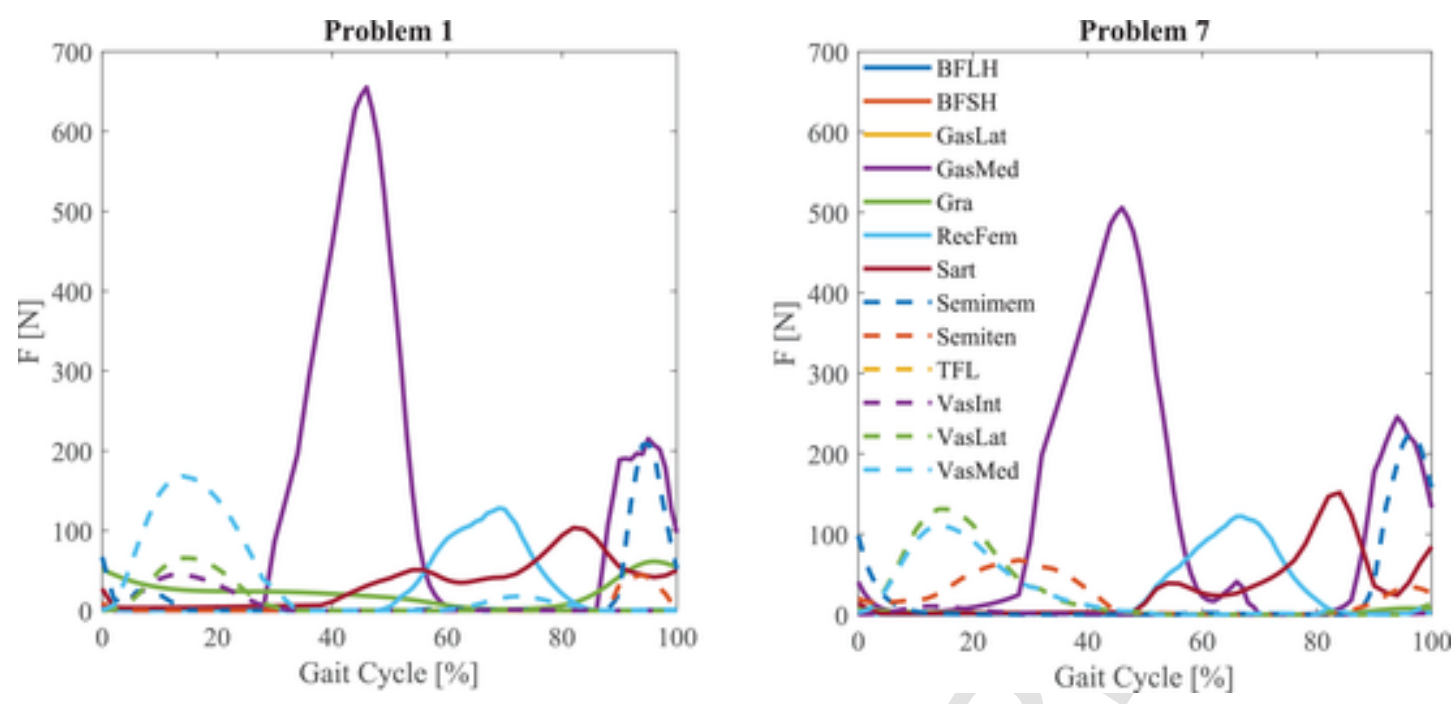

Fig. 6. Muscle contributions to medial contact force of a calibration trial in Problems 1 and 7.
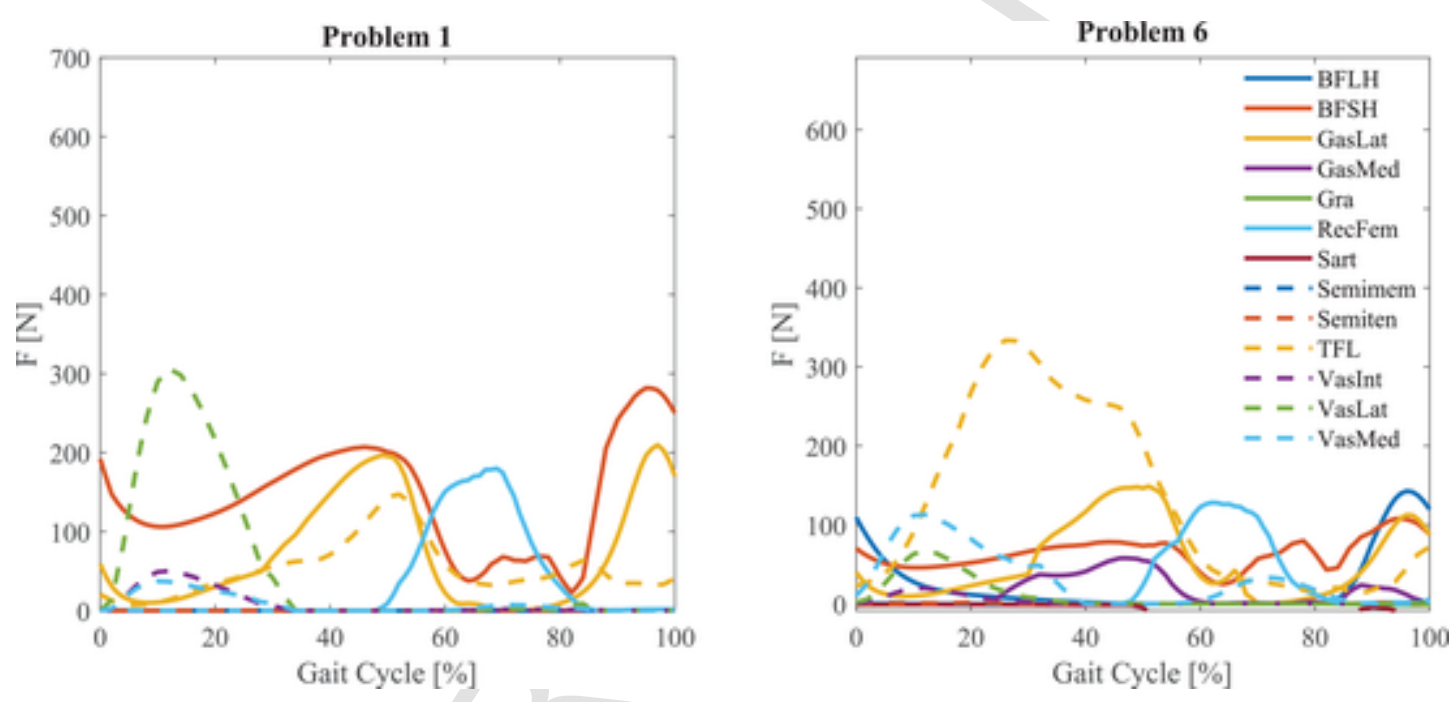

Fig. 7. Muscle contributions to lateral contact force of a calibration trial in Problems 1 and 6.

was the recent work reported by Navacchia and co-workers [29]. Using a scaled generic musculoskeletal model with 20 leg muscles, the authors concluded that maximum isometric force, muscle lines of action, and joint kinematics had the greatest influence on total knee contact force and varus-valgus contact moment, while optimal muscle fiber length and tendon slack length (calculated through pennation angle and optimal fiber length) had little influence. Variability in model outputs spanned experimental benchmark measurements well for total knee contact force but not for varus-valgus contact moment, suggesting that inaccurate medial and lateral knee contact forces would have been predicted in that study. In contrast, our study used a musculoskeletal model with subject-specific bone geometry, 44 leg muscles, and subject-specific fluoroscopic knee kinematics and concluded that optimal muscle fiber length, tendon slack length, and moment arm offsets were important for predicting medial and lateral knee contact force accurately. Thus, only the conclusions regarding moment arm offset parameters are consistent with the conclusions of Navacchia et al. Maximum isometric force was not identified in our study because it is redundant with muscle activation amplitude for determination of active muscle force, and muscles in our model produced little passive force. Inconsistencies between the two studies in identified parameters may be due to differences in model construction and kinematic inputs as well as differences in study goals (i.e., analysis of knee contact force sensitivity to individual parameter values, which does not guarantee that accurate knee contact forces can be achieved, versus evaluation of which parameters should be calibrated to predict accurate knee contact forces). Overall, our study suggests that if three types of model parameter values are well calibrated, a traditional musculoskeletal model can predict both medial and lateral knee contact force with good accuracy.

Other studies, which did not consider moment arm variations, concluded that tendon slack lengths played a more important role than optimal muscle fiber lengths when estimating muscle forces via optimization methods [45]. De Groote et al. [46] observed that tendon slack lengths generally had more influence than optimal fiber lengths on estimated muscle forces, though some muscle forces were sensitive to optimal fiber length values as well. In our study, lateral knee contact force was already estimated accurately without calibrating optimal fiber lengths (Problem 6). When optimal fiber lengths were included as design variables (Problem 7), prediction of medial contact force improved slightly, while prediction of lateral contact force worsened.

For all of our optimization problem formulations, errors in predicted medial and lateral knee contact forces were of similar or lower magnitude compared to other studies $[5,47,48]$. Though experimental knee contact force information was used to calibrate model parameter values (outer level optimization), no knee contact force information was used when estimating muscle activations (inner level optimiza- 

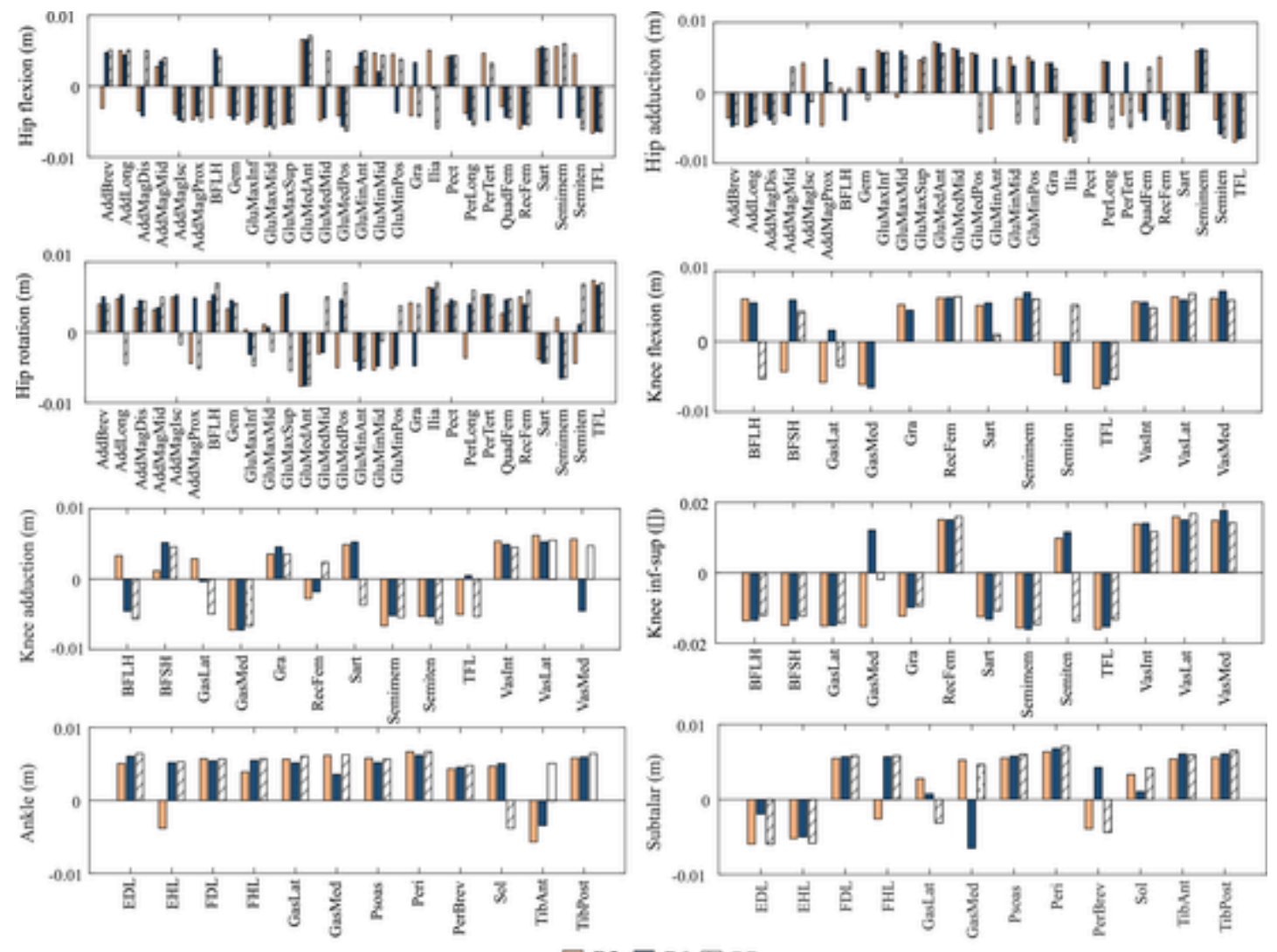

P6 $\square$ P7

Fig. 8. Moment arm deviations for Problems 5, 6, and 7. Note that knee inf-sup "moment arm" is dimensionless.

tion). Calibrated model parameter values produced muscles that operated within physiological ranges [49] in all nine optimization problems. We observed that when moment arm deviations were not included as design variables, the second peak of medial contact force at about $50 \%$ of the gait cycle was overpredicted, as observed in other studies that did not calibrate this parameter $[15,44]$. These findings suggest that existing musculoskeletal model formulations are capable of predicting knee contact forces accurately as long as critical model parameter values are calibrated properly.

Differences in estimated knee contact forces can be explained by variations in a few muscle parameter values, which in turn are responsible for different muscle contributions to knee contact force [50]. Overprediction of the second peak of medial force at about $50 \%$ of the gait cycle, especially in problems where no moment arm deviations were optimized, was due primarily to different GasMed force contributions (Fig. 6). The unrealistic peak of lateral force obtained at about $10 \%$ of the gait cycle in several formulations was due to different BFSH, GasLat, TFL, and GasMed force contributions (Fig. 7). As mentioned above, the most important design variables were moment arm deviations (Fig. 8), which were within a physiological range $(<1 \mathrm{~cm})$ for all muscles in all problems. However, these small changes affected the optimizations enough to produce the differences in knee contact force predictions mentioned above.

Additionally, we tested two common issues in studies dealing with muscle and knee contact force prediction. The first issue was that lateral contact forces were tracked less accurately than were medial contact forces [5]. We ran an extra optimization problem where we increased the weight on lateral force tracking by $10 \%$ and decreased the weight on medial force tracking by $10 \%$. This change improved lateral contact force tracking for calibration trials, but prediction of both medial and lateral contact forces for prediction trials was worse than when both forces were weighted equally. Obtaining accurate lateral con- tact force predictions remains challenging. More accurate models of the knee, for example including the lateral collateral ligament (which was not included in our study), may lead to better lateral contact force predictions. The second issue was whether scaling optimal muscle fiber lengths and tendon slack lengths equally led to comparable results, since the observed variations in optimal fiber lengths and tendon slack lengths were low. However, the results were indeed better when calibration of these two types of parameters were decoupled, consistent with the work of other groups [23].

Nevertheless, a proper calibration of musculo-tendon parameters that leads to accurate knee contact forces remains unknown when no knee contact force information is available. However, the results of this study give insight into which parameters should be calibrated to obtain accurate medial and lateral knee contact forces, as well as what are the main muscle force differences obtained when using different sets of design variables.

A drawback of using non-linear optimization (in our case, in the outer level) was the fact that the optimization algorithm could experience entrapment in local minima, and thus we could not guarantee that solutions of the two-level optimizations were global minima. However, we are confident that the muscle activation solutions were global minima given a set of musculoskeletal parameters (inner-level optimization), since these solutions were obtained using a quadratic programming algorithm and the problem being solved was convex. To address the local minima issue, we systematically started each outer level optimization from different initial guesses. We chose the solutions that estimated medial and lateral contact force magnitudes the best, which also had the best shape estimates.

This study had several limitations that should be taken into account. Apart from optimal muscle fiber lengths, tendon slack lengths, and moment arm deviations, other musculoskeletal model parameters could be calibrated and could influence muscle and contact force prediction. 
For example, parameters related to the normalized muscle force-length curve could also be varied [51]. However, the chosen parameters were the ones that have been shown to have the greatest influence on muscle force estimation [45], and they are also parameters amenable to calibration through EMG-driven modeling methods [16-19]. Maximum isometric forces were not included in the set of design variables since they could introduce numerical redundancies with muscle activations and moment arms. To estimate maximum isometric forces, we recommend using the scaling methods reported by Handsfield et al. [52]. As we did an extensive set of analyses in this study, we performed our evaluation using experimental data from a single 88-year old subject. Although our single-subject design is a limitation, future work could expand on the promising approaches from this study with instrumented knee datasets from other subjects available at http://simtk.org/projects/kneeloads. In addition, our results cannot be easily applied to datasets without in vivo contact force measurements. We expect that dynamic variables, such as joint moments, muscle forces, and knee contact forces, would be different in other subjects, which would in turn require different model parameter values. However, we expect the influence on the solution of each analyzed parameter type to follow a similar trend, since the optimization problem formulation to estimate knee contact forces would be the same. It is not known if the results of the analysis presented here would be similar if non-walking movements were used to assess the calibration approach. In the future, it would be valuable to repeat the evaluation for other motions such as crouch gait, squatting, or stair climbing to evaluate such differences.

In conclusion, this study demonstrated that medial and lateral knee contact forces can be estimated accurately using an existing musculoskeletal model structure as long as model parameter values are properly calibrated. The most accurate estimates of lateral knee contact forces, which are the most difficult to match, were obtained when optimizing tendon slack lengths and moment arm deviations. In contrast, the most accurate estimates of medial knee contact forces occurred when optimal muscle fiber lengths, tendon slack lengths, and moment arm deviations were adjusted simultaneously. These conclusions should be taken into account when selecting the set of musculo-tendon parameters to calibrate when solving muscle-force sharing problems depending on the purpose of the study. Based on our results, we suggest calibrating only tendon slack lengths and moment arms, since problem formulations that calibrated these parameters lead to the overall best predictions of both medial and lateral contact forces. A challenge for the future will be finding ways to calibrate these model parameter values when in vivo knee contact force measurements are not available.

\section{Declaration of Competing Interest}

None.

\section{Acknowledgments}

Gil Serrancolí acknowledges support from the Serra Húnter Program.

\section{Funding}

None.

\section{Ethical approval}

Not required.

\section{Supplementary materials}

Supplementary material associated with this article can be found, in the online version, at doi:10.1016/j.medengphy.2020.09.004.

\section{References}

[1] EUMUSC: European musculoskeletal conditions surveillance and information network (2019, December 1). Musculoskeletal Health Europe. Retreived from www.eumusc.net

[2] J.W.J. Bijlsma, F. Berenbaum, F.P.J.G. Lafeber Osteoarthritis: an update with relevance for clinical practice. Lancet 2011;377:2115-2126. doi:10.1016/ S0140-6736(11)60243-2.

[3] R.F. Loeser Aging processes and the development of osteoarthritis. Curr Opin Rheumatol 2013;25:108-113. doi:10.1097/BOR.0b013e32835a9428.

[4] Y. Bei, B.J. Fregly Multibody dynamic simulation of knee contact mechanics. Med Eng Phys 2004;26:777-789. doi:10.1016/j.medengphy.2004.07.004.

[5] A.L. Kinney, T.F. Besier, D.D. D'Lima, B.J. Fregly Update on grand challenge competition to predict in vivo knee loads. J. Biomech Eng 2013;135(2):021012. doi:10.1115/1.4023255.

[6] B.J. Fregly, T.F. Besier, D.G. Lloyd, S.L. Delp, S.A. Banks, M.G. Pandy, et al. Grand challenge competition to predict in vivo knee loads. J Orthop Res 2012;30:503-513. doi:10.1002/jor.22023.

[7] I. Kutzner, B. Heinlein, F. Graichen, A. Bender, A. Rohlmann, A. Halder, et al. Loading of the knee joint during activities of daily living measured in vivo in five subjects. J Biomech 2010;43:2164-2173. doi:10.1016/ j.jbiomech.2010.03.046.

[8] D.D. D'Lima, C.P. Townsend, S.W. Arms, B.A. Morris, C.W. Colwell An implantable telemetry device to measure intra-articular tibial forces. J Biomech 2005;38:299-304. doi:10.1016/j.jbiomech.2004.02.011.

[9] W.R. Taylor, P. Schütz, G. Bergmann, R. List, B. Postolka, M. Hitz, et al. A comprehensive assessment of the musculoskeletal system: the CAMS-Knee data set. J Biomech 2017;65:32-39. doi:10.1016/j.jbiomech.2017.09.022.

[10] W. Herzog, D. Longino, A. Clark The role of muscles in joint adaptation and degeneration. Langenbeck's Arch. Surg. 2003;388:305-315. doi:10.1007/ s00423-003-0402-6.

[11] D.G. Thelen, F.C. Anderson, S.L. Delp Generating dynamic simulations of movement using computed muscle control. J Biomech 2003;36:321-328. doi:10.1016/S0021-9290(02)00432-3.

[12] K. Sasaki, R.R. Neptune Muscle mechanical work and elastic energy utilization during walking and running near the preferred gait transition speed. Gait Posture 2006;23:383-390. doi:10.1016/j.gaitpost.2005.05.002.

[13] Y. Lin, J.P. Walter, S.A. Banks, M.G. Pandy, B.J. Fregly Simultaneous prediction of muscle and contact forces in the knee during gait. J Biomech 2010;43:945-952. doi:10.1016/j.jbiomech.2009.10.048.

[14] S.R. Hamner, A. Seth, S.L. Delp Muscle contributions to propulsion and support during running. J Biomech 2010;43:2709-2716. doi:10.1016/ j.jbiomech.2010.06.025.Muscle.

[15] M.A. Marra, V. Vanheule, R. Fluit, B.H.F.J.M. Koopman, J. Rasmussen, N. Verdonschot, et al. A subject-specific musculoskeletal modeling framework to predict in vivo mechanics of total knee arthroplasty. J Biomech Eng 2015;137:1-12. doi:10.1115/1.4029258.

[16] D.G. Lloyd, T.F. Besier An EMG-driven musculoskeletal model to estimate muscle forces and knee joint moments in vivo. J Biomech 2003;36:765-776. doi:10.1016/S0021-9290(03)00010-1.

[17] K. Manal, T.S. Buchanan An electromyogram-driven musculoskeletal model of the knee to predict in vivo joint contact forces during normal and novel gait patterns. J Biomech Eng 2013;135:021014. doi:10.1115/1.4023457.

[18] M. Sartori, M. Reggiani, D. Farina, D.G. Lloyd EMG-driven forward-dynamic estimation of muscle force and joint moment about multiple degrees of freedom in the human lower extremity. PLoS ONE 2012;7:e52618. doi:10.1371/ journal.pone.0052618.

[19] A.J. Meyer, C. Patten, B.J. Fregly Lower extremity EMG-driven modeling of walking with automated adjustment of musculoskeletal geometry. PLoS ONE 2017;12:e0179698.

[20] M. Zuk, M. Syczewska, C. Pezowicz Influence of uncertainty in selected musculoskeletal model parameters on muscle forces estimated in inverse dynamics-based static optimization and hybrid approach. J Biomech Eng 2018;140:121001. doi:10.1115/1.4040943.

[21] C.A. Myers, P.J. Laz, K.B. Shelburne, B.S. Davidson A probabilistic approach to quantify the impact of uncertainty propagation in musculoskeletal simulations. Ann Biomed Eng 2015;43:1098-1111. doi:10.1007/s10439-014-1181-7.

[22] C. Redl, M. Gfoehler, M.G. Pandy Sensitivity of muscle force estimates to variations in muscle-tendon properties. Hum Mov Sci 2007;26:306-319. doi:10.1016/j.humov.2007.01.008.

[23] C.R. Winby, D.G. Lloyd, T.B. Kirk Evaluation of different analytical methods for subject-specific scaling of musculotendon parameters. J Biomech 2008;41:1682-1688. doi:10.1016/j.jbiomech.2008.03.008.

[24] M. Xiao, J. Higginson Sensitivity of estimated muscle force in forward simulation of normal walking. J. Appl. Biomech. 2010;26:142-149.

[25] L.L. Menegaldo, L.F. Oliveira The influence of modeling hypothesis and experimental methodologies in the accuracy of muscle force estimation using EMG-driven models. Multibody Syst. Dyn. 2012;28:21-36. doi:10.1007/ s11044-011-9273-8.

[26] A.Van Campen, G. Pipeleers, F. De Groote, I. Jonkers, J. De Schutter A new method for estimating subject-specific muscle - tendon parameters of the knee joint actuators: a simulation study. Int J Numer Method Biomed Eng 2014;30:969-987. doi:10.1002/cnm.2639.

[27] D.C. Ackland, Y.-.C. Lin, M.G. Pandy Sensitivity of model predictions of muscle function to changes in moment arms and muscle-tendon properties: a Monte-Carlo analysis. J Biomech 2012;45:1463-1471. doi:10.1016/ j.jbiomech.2012.02.023. 
[28] G. Valente, L. Pitto, D. Testi, A. Seth, S.L. Delp, R. Stagni, et al. Are subject-specific musculoskeletal models robust to the uncertainties in parameter identification? PLoS ONE 2014;9:e112625. doi:10.1371/journal.pone.0112625.

[29] A. Navacchia, C.A. Myers, P. Rullkoetter, K.B. Shelbourne Prediction of in vivo knee joint loads using a global probabilistic analysis. J Biomech Eng 2016;138:4032379. doi:10.1115/1.4032379.

[30] M.E. Lund, M.S. Andersen, M. de Zee, J. Rasmussen Scaling of musculoskeletal models from static and dynamic trials. Int Biomech 2015;2:1-11. doi:10.1080/ 23335432.2014.993706.

[31] M.O. Heller, W.R. Taylor, C. Perka, G.N. Duda The influence of alignment on the musculo-skeletal loading conditions at the knee. Langenbeck's Arch Surg 2003;388:291-297. doi:10.1007/s00423-003-0406-2.

[32] S.L. Delp, F.C. Anderson, A.S. Arnold, P. Loan, A. Habib, C.T. John, et al. OpenSim: open-source software to create and analyze dynamic simulations of movement. IEEE Trans Biomed Eng 2007;54:1940-1950. doi:10.1109/ TBME.2007.901024.

[33] A. Seth, J.L. Hicks, T.K. Uchida, A. Habib, C.L. Dembia, J.J. Dunne, et al. OpenSim: simulating musculoskeletal dynamics and neuromuscular control to study human and animal movement. PLoS Comput Biol 2018;14:e1006223. doi:10.1371/journal.pcbi.1006223.

[34] G. Serrancoli, A.L. Kinney, B.J. Fregly, J.M. Font-Llagunes Neuromusculoskeletal model calibration significantly affects predicted knee contact forces for walking. J Biomech Eng 2016;138:081001. doi:10.1115/ 1.4033673 .

[35] E.M. Arnold, S.R. Hamner, A. Seth, M. Millard, S.L. Delp How muscle fiber lengths and velocities affect muscle force generation as humans walk and run at different speeds. J Exp Biol 2013;216:2150-2160. doi:10.1109/20.619708.

[36] E.M. Arnold, S.R. Ward, R.L. Lieber, S.L. Delp A model of the lower limb for analysis of human movement. Ann Biomed Eng 2010;38:269-279. doi:10.1007/ s10439-009-9852-5.

[37] J.P. Walter, A.L. Kinney, S.A. Banks, D.D. D'Lima, T.F. Besier, D.G. Lloyd, et al. Muscle synergies may improve optimization prediction of knee contact forces during walking. J Biomech Eng 2014;136:021031. doi:10.1115/1.4026428.

[38] D. Zhao, S.A. Banks, D.D. D’Lima, C.W. Colwell Jr., B.J. Fregly In vivo medial and lateral tibial loads during dynamic and high flexion activities. J Orthop Res 2007;25:593-602. doi:10.1002/jor.20362.

[39] T.F. Coleman, Y. Li An interior trust region approach for nonlinear minimization subject to bounds. SIAM J Optim 1994;6:418-445. doi:10.1137/ 0806023.

[40] E.M. Arnold, S.L. Delp Fibre operating lengths of human lower limb muscles during walking. Philos Trans R Soc Lond B Biol Sci 2011;366:1530-1539. doi:10.1098/rstb.2010.0345.

[41] F. De Groote, A.L. Kinney, A.V. Rao, B.J. Fregly Evaluation of direct collocation optimal control problem formulations for solving the muscle redundancy problem. Ann Biomed Eng 2016;44:2922-2936. doi:10.1007/ s10439-016-1591-9.

[42] T.A. Correa, R. Baker, H. Kerr Graham, M.G. Pandy Accuracy of generic musculoskeletal models in predicting the functional roles of muscles in human gait. J Biomech 2011;44:2096-2105. doi:10.1016/j.jbiomech.2011.05.023.

[43] L. Scheys, A. Spaepen, P. Suetens, I. Jonkers Calculated moment-arm and muscle-tendon lengths during gait differ substantially using MR based versus rescaled generic lower-limb musculoskeletal models. Gait Posture 2008;28:640-648. doi:10.1016/j.gaitpost.2008.04.010.

[44] L. Modenese, E. Montefiori, A. Wang, S. Wesarg, M. Viceconti, C. Mazzà Investigation of the dependence of joint contact forces on musculotendon parameters using a codified workflow for image-based modelling. J Biomech 2018;73:108-118. doi:10.1016/j.jbiomech.2018.03.039.

[45] C.Y. Scovil, J.L. Ronsky Sensitivity of a hill-based muscle model to perturbations in model parameters. J Biomech 2006;39:2055-2063. doi:10.1016/j.jbiomech.2005.06.005.

[46] F. De Groote, A. Van Campen, I. Jonkers, J. De Schutter Sensitivity of dynamic simulations of gait and dynamometer experiments to hill muscle model parameters of knee flexors and extensors. J Biomech 2010;43:1876-1883. doi:10.1016/j.jbiomech.2010.03.022.

[47] R.E. Richards, M.S. Andersen, J. Harlaar, J.C. van den Noort Relationship between knee joint contact forces and external knee joint moments in patients with medial knee osteoarthritis: effects of gait modifications. Osteoarthr. Cartil. 2018;26:1203-1214. doi:10.1016/j.joca.2018.04.011.

[48] F. Schellenberg, W.R. Taylor, A. Trepczynski, R. List, I. Kutzner, P. Schütz, et al. Evaluation of the accuracy of musculoskeletal simulation during squats by means of instrumented knee prostheses. Med Eng Phys 2018;61:95-99. doi:10.1016/j.medengphy.2018.09.004.

[49] E.M. Arnold, S.L. Delp Fibre operating lengths of human lower limb muscles during walking. Philos Trans R Soc B Biol Sci 2011;366:1530-1539. doi:10.1098/rstb.2010.0345.

[50] C.R. Winby, D.G. Lloyd, T.F. Besier, T.B. Kirk Muscle and external load contribution to knee joint contact loads during normal gait. J Biomech 2009;42:2294-2300. doi:10.1016/j.jbiomech.2009.06.019.

[51] Z.J. Domire, J.H. Challis A critical examination of the maximum velocity of shortening used in simulation models of human movement. Comput Methods Biomech Biomed Engin 2010;13:693-699. doi:10.1080/10255840903453082.

[52] G.G. Handsfield, C.H. Meyer, J.M. Hart, M.F. Abel, S.S. Blemker Relationships of 35 lower limb muscles to height and body mass quantified using MRI. J Biomech 2014;47:631-638. doi:10.1016/j.jbiomech.2013.12.002. 